\title{
16 Sind Alpert von Metz und der Pseudo-Alkuin frühe Zeugen der altfranzösischen Epik?
}

\begin{abstract}
Résumé : Selon Menéndez Pidal, non seulement une observation d'Albert « de Metz » (entre 1021 et 1025) relative à des cantilenae narratives, mais encore le renvoi, dans le ms. Paris B. N. lat. 5354 (vers 1050 ou avant), à la Vita Karoli d'un mystérieux (Pseudo-)Alcuin, laquelle aurait contenu des motifs épiques, témoigneraient de l'existence de chansons de geste. Pourtant, Albert est probablement un Néerlandais ; en tout cas, il vit depuis longtemps aux Pays-Bas lorsqu'il formule l'observation en question dans une lettre à l'évêque Burchard de Worms, natif de Hesse. La remarque d'Albert ne semble donc pas porter sur la littérature française. En outre, contrairement à ce que croyait Menéndez Pidal, une Vita Karoli faussement attribuée à Alcuin a survécu dans plusieurs manuscrits, mais se trouve être simplement celle d'Éginhard, et supposer un manuscrit de cette dernière enrichi de motifs épiques serait une hypothèse dépourvue de tout parallèle. Le renvoi, dans le ms. 5354, à un Pseudo-Alcuin "épicophile », s'il semble donc infondé, renferme toutefois une subordonnée qui prouve que le scribe connaissait, indépendamment de toute Vita Karoli, des chansons françaises relatives à Charlemagne et fondées sur des motifs presque sûrement fictifs. D'après l'étude la plus récente, le manuscrit, y compris la remarque, remonterait au premier tiers du $\mathrm{XI}^{\mathrm{e}}$ siècle. Ce serait donc le plus ancien témoignage d'une activité épique centrée, dans une langue romane, sur la personne de Charlemagne.
\end{abstract}

Im Jahr 2009 ist gerade ein halbes Jahrhundert vergangen, seit Menéndez Pidal eine Bemerkung Alperts von Metz zu einem Eckstein seiner Theorie über die Entstehung der altfranzösischen Epik machte und zudem eine Vermutung über einen Pseudo-Alkuin als ebenfalls frühen Zeugen dieser Epik aussprach. In den darauf folgenden Jahren hätte man es einem jungen mediävistisch engagierten Romanisten sehr verübelt, wenn er zumindest Alperts Bemerkung nicht gekannt hätte. Doch die große Kontroverse zwischen ,Neotraditionalisten‘ und ,Individualisten` verebbte allmählich, ohne Sieg einer Partei, ja auch ohne klaren Kompromiss. So siedelten sich die meisten jungen Forscher nicht erst in diesem Gebiet an; denn dort schien die Grenze des Wissbaren erreicht. Ich gestehe, dass ich diese Anschauung nicht teile. Man kann hier durchaus noch wissenswerte Fakten zu Tage fördern, und zwar keineswegs immer zugunsten derselben Partei. Das sei hier zu zeigen versucht.

Die Bemerkung, welche Alpert / Albert ${ }^{1}$ von Metz über zu seiner Zeit gesungene cantilenae im Widmungsbrief zu seinem zeitgeschichtlichen Werk De di-

1 Die essentiell einzige Handschrift der beiden ihm mit Sicherheit zugehörigen Werke (vgl. Anm. 5) hat Alpertus mit - $p$ - an den drei Vorkommensstellen des Namens, nämlich in Alperts

Anmerkung: Erstmals veröffentlicht in: Romanische Forschungen 121 (2009), 477-495.

Ә Open Access. (C 2019 Gustav Adolf Beckmann, publiziert von De Gruyter. (c))BY-NC-ND Dieses Werk ist lizenziert unter der Creative Commons Attribution-NonCommercial-NoDerivatives 4.0 Lizenz.

https://doi.org/10.1515/9783110615692-016 
versitate temporum nach dem 5. Juni 1021 und spätestens einige Wochen vor dem 20. August $1025^{2}$ niederschrieb, ist der gelehrten Welt zugänglich, seit erstmalig J. G. Eccard (1723), dann vor allem Pertz in den Monumenta Germaniae Historica (1841) die beiden mit Autornamen auf uns gekommenen Werke Alperts $^{3}$ edierte (nämlich außer dem genannten Werk einen ebenfalls zeitgeschichtlichen Bericht über die letzten Lebensjahre des Bischofs Dietrich I. von Metz, † 984). ${ }^{4}$ Doch wurde die Bemerkung von der romanistischen Forschung erst sehr spät wahrgenommen, so weder von Gaston Paris (1865, 21905), Rajna (1884) oder Bédier (1904-1908, 31926-1929) noch von Faral in seiner auf Vollständigkeit der Zeugnisse angelegten Pariser Doktorthese über die Jongleure in

Selbstnennung am Ende seines Berichts über Bischof Dietrich sowie in der Antwort des Widmungsempfängers und im Incipit des eigentlichen Textes von De diversitate (van Rij 1980: 4, 6, 120); ebenso haben - $p$ - eine Metzer Erwähnung der Historia Alperti aus dem 11. Jahrhundert (van Rij 1980: XII), von neueren Autoren dann Manitius (1923: 278-283 passim), der Romanist Favati (1960: 97) und die Historiker (das LM, Art. Albert [13], allerdings nur als Zweitform). Doch schon Sigebert von Gembloux, der durch seinen zwanzigjährigen Aufenthalt in Metz (um 1050 bis etwa 1071) mit Alperts Werken bestens vertraut war, spricht in seinem Catalogus de viris illustribus (ed. Witte 1974: 92) von Albertus (!) monachus Mettensis (van Rij 1980: IX, XII, XIX, XXXI, XLVIII, L); ihm folgen die Romanisten (mit Ausnahme von Favati) und das LM in seiner Lemmaform. Geht man von ,Albert‘ aus, so könnte Alpert oberdeutsch erscheinen. Aber Alperts Leben und Schriften weisen keinerlei Beziehungen zu Oberdeutschland auf (s. weiter unten passim), und auch der Schreibort Metz liegt dem mitteldeutschen (moselfränkischen und westpfälzischen) Sprachgebiet näher als dem oberdeutschen. Ich vermute deshalb, dass es sich ursprünglich um den Namen Ald-bert handelte; auch Foerstemann 1900 (ohne Korrektur bei Kauffmann 1968) bringt ja ein Olpert unter ALDA- ,alt' unter. Der Name hätte dann im ersten Glied Auslautverhärtung erfahren (wie sie im altsächsischen Namengut schon im 9. Jahrhundert gut bezeugt ist, vgl. Alt-, Gunt-, Thiat- u. ä. bei Schlaug 1962 s. vv., und im Niederld. im 10. Jahrhundert durchdrang, van Loey § 47); es folgten progressive Assimilation und Fall des mittleren Konsonanten (Schlaug 1955 s. vv. belegt Thiapbalt 1024, Liupraht 1042/63). Doch in der Romania haben wohl schon Alperts Klosterbrüder, spätestens Sigebert, dann seine Abschreiber den Namen identifiziert mit dem weit häufigeren Namen Albert.

2 Die Datierung ist unstrittig und unbestreitbar, vgl. die Begründung bei van Rij 1980: XIV. Da die Widmung in größerer Entfernung von Worms geschrieben ist, aber von ihrem Adressaten Bischof Burchard von Worms noch beantwortet wurde (van Rij 1980: 4 f.), muss sie spätestens einige Wochen vor dessen Tod entstanden sein. Ein wenigstens wahrscheinlicher terminus ad quem ist schon der 13. Juli 1024, Todestag Heinrichs II. (van Rij 1980: XIV). - Ich ziehe im Folgenden van Rijs Einleitung zur zweiten Auflage seiner Übersetzung (1999: 9-42) nur vereinzelt dort heran, wo sie wesentlich über die Einleitung zur Ausgabe (1980: IX-LIX) hinausgeht. 3 Über die anonymen, ihm wahrscheinlich als drittes Werk zuzuweisenden Miracula Walburgae Tielensia vgl. weiter unten.

4 Spätere Ausgaben beider Werke: Migne (1853, nach Pertz), Dederich (1859, unzuverlässig, mit dt. Übersetzung), Pijnacker Hordijk (1908, Faksimile), van Rij (1980, mit niederld. Übersetzung, die mit überarbeiteter Einleitung 1999 in 2. Aufl. erschien); nur De diversitate temporum: Hulshof (1916). 
Frankreich (1909, $\left.{ }^{2} 1971\right)$. Wohl aber gab Manitius in seiner Geschichte der mittellateinischen Literatur die Stelle gerafft in indirekter Rede wieder (1923: 280); Alpert verteidigt sich im Voraus gegen mögliche Kritiker: „Und wenn man ihm vorwerfe, dass er längst Bekanntes geschrieben habe, so antworte er, dass man das Bekannte gern öfter höre, wie es bei den Volksgesängen (cantilenis) sei, deren alte wegen zu häufiger Wiederholung anwiderten, während man die neuen gern recht oft anhöre." Sehen wir uns genauigkeitshalber den etwas verschlungeneren Urtext an (ed. van Rij 1980: 4):

„Et si demum hęc obicientur et arguar, quod omnibus cognita scripserim, tuo consilio perpendant me hac responsione uti: nota delectabiliter sepius audiri, ut solet fieri in cantilenis, quod, veteribus ex assiduitate fastiditis, novę frequentius in dies repetitę delectabilius audiuntur.“5

In möglichst enger deutscher Übersetzung:

Und wenn [mir] schließlich Folgendes entgegengehalten wird und ich getadelt werde, dass ich [nur] allen [schon] bekannte Dinge niedergeschrieben hätte, so seien sie [scil. jene Kritiker] auf deinen Rat hin ernsthaft zu überdenken aufgefordert, dass ich [immerhin] diese Antwort parat habe: [Auch] bekannte Dinge lassen sich mit Genuss häufiger hören, wie es mit den Kantilenen zu gehen pflegt, dass [nämlich], wenn [einem] die alten durch übermäßige Vertrautheit leid geworden sind, man doch die neuen, selbst wenn sie bald täglich wiederholt werden, recht gern hört.

In die Romanistik scheint die Stelle erst 1951 einzuziehen, zwar gleich doppelt, doch zunächst in wenig glücklicher Weise. Aus Manitius hat sie Sicilano (1951: 34 Anm. 1). Er beschränkt sich auf einen Satz innerhalb einer Fußnote: «Et il y a peu de choses à tirer du passage d'Albert de Metz (cité par Manitius, Geschichte, II, 282) qui semble nous dire que, une fois mortes les vieilles cantilènes, on écoute avec un plaisir plus grand les nouvelles sans cesse répétées.» Dieser Satz steht ohne Zeitangabe hinter einer Erwähnung des Thibaut von Vernon, der «vers 1040» wirkte, verharmlost also Alperts Bemerkung schon durch Suggestion eines zu späten Zeitpunktes. Ferner sind nach dem Urtext, den Sici-

5 Der Text hat keine Varianten; denn Alperts Werke sind im Wesentlichen nur in einer Sammelhandschrift des späten 11. Jahrhunderts erhalten (heute Niedersächsische Landesbibliothek XII B 712a, vorher Wolfenbüttel, geschrieben so gut wie sicher in Metz); vgl. van Rij 1980: XLV-L und Abbildung nach VII. Dass die kleine in De diversitate eingebaute Vita des Bischofs Ansfried von Utrecht aus einer heute verlorenen Utrechter Handschrift von den Bollandisten abgedruckt wurde und dass eine heute Florentiner Handschrift aus dem späten 11. Jahrhundert jene Polemik zwischen einem zum Judentum Konvertierten und einem Priester aus De diversitate ausgeschrieben hat, die Alpert dort anhangsweise aufführt (van Rij 1980: L-LII), braucht uns nicht zu interessieren. 
liano nicht zitiert, die alten cantilenae nicht schon irgendwann «abgestorben, sondern erzeugen noch Überdruss; indem Siciliano so den zeitlichen Zusammenhang zwischen Alt und Neu lockert, tritt die Kontinuität der von Alpert angedeuteten Tradition weniger hervor. Vor allem aber fehlt die Hauptsache; denn die Erwähnung der cantilenae ist doch Teil eines Vergleichs. Laut Alpert sollen seine Leser einen und denselben (sehr detailreichen historischen) Erzählstoff, den sie schon kennen, nunmehr, durch ihn selbst und damit zwangsläufig in variierter Perspektive dargeboten, durchaus ein zweites Mal goutieren können so wie es auch bei den cantilenae der Fall sei. Damit dieser Vergleich auch nur im Geringsten sinnvoll ist, müssen die alten wie die neuen cantilenae ebenfalls Erzählstoff bieten (also ,episch', nicht ,lyrisch` sein), und zwar müssen jeweils (zumindest) eine alte und eine neue prinzipiell einen und denselben Erzählstoff bieten, nur wiederum mit einer gewissen Variation. Und gerade die Tatsache, dass Alpert diese cantilenae nicht in irgend einem indifferenten Zusammenhang, sondern in einer Argumentation pro domo als Vergleichsobjekte heranzieht, zeigt, dass er nicht nur die Bekanntschaft mit einer Mehrzahl solcher cantilenae, sondern auch die wenigstens prinzipielle Freude an ihnen und wohl auch die Duldung kleinerer inhaltlicher Unterschiede der jeweils neuen gegenüber den alten als das Normale voraussetzt. ${ }^{6}$ Festhalten sollte man noch, dass in Alperts Formulierung diese Normal-Hörer (‘audires kommt zweimal vor) zwar Überdruss oder Gefallen empfinden, aber nicht selbst die Singenden, die «Repetierenden>, zu sein brauchen. Zusammenfassend gesagt, scheint also Alperts Vergleich sehr wohl eine frühe und schon recht populäre Kantilenen-Epik zu bezeugen, doch wohlgemerkt ohne dass man über deren Sprache, ihre Erzählstoffe und den Umfang der einzelnen «Werke〉 bzw. Darbietungen Auskunft erhält.

Doch fahren wir fort mit der Forschungsgeschichte! Kaum glücklicher als Siciliano verfuhr gleichzeitig Jules Horrent, ebenfalls nur in einer Fußnote und ohne Nennung des Vergleichszusammenhangs (1951: 232 Anm. 2). Er war durch den Nederlandisten van Mierlo (1935: 76 f.) auf Alperts Passus aufmerksam geworden, datierte ihn aber versehentlich «vers 1080», wodurch er «pratiquement tout intérêt» ${ }^{7}$ verlor und acht weitere Jahre in seinem Dornröschenschlaf ver-

\footnotetext{
6 Auch 17 Jahre später hatte sich an Sicilianos Haltung nichts geändert: Jetzt in offener Polemik gegen Menéndez Pidal befindlich, hatte er wieder nur einen Teil einer Fußnote übrig (1968: 59 Anm., Punkt 3), um Alperts Passus „assez obscur“ zu finden, seinen Vergleichscharakter erneut zu ignorieren und deshalb summarisch sagen zu können, Alpert präzisiere nicht „de quel genre de cantilènes il s’agit.“ Daran schließt sich der Verweis an: „Sur le caractère aventureux des interprétations de M. Menéndez Pidal, cf. S. Pellegrini, Studi rolandiani e trobadorici, 1964“ - doch Pellegrini erwähnt Alpert von Metz nicht.
}

7 So rückblickend Lecoy (1959: 114) zu Horrents Anmerkung. 
harrte. Immerhin lernte aus Horrents Fußnote Ramón Menéndez Pidal die Bemerkung Alperts kennen. Im Jahr 1959 kommentierte er sie dann mit ihrem richtigen Datum in La Chanson de Roland y el neotradicionalismo. ${ }^{8}$ Unmittelbar nach der Lektüre dieses Buches befasste sich aus den Reihen seiner Opponenten wenigstens ${ }^{9}$ Félix Lecoy mit der Stelle, wie sein Diskussionsbeitrag vom 23. Juli 1959 auf dem Kogress der Société Rencesvals in Poitiers zeigt (Lecoy 1960: 114); wesentlich genauer ging er auf Alperts Bemerkung dann ein in seiner 46-seitigen Rezension (1963: 98-133, hier 121f.) der französischen Ausgabe von Menéndez Pidals Buch. Interessanterweise verlief die Diskussion beider Forscher nur teilweise kontrovers.

Menéndez Pidal gibt dem Wort cantilenae bei Alpert kurzerhand dieselbe Bedeutung, die es 100 Jahre später etwa in William of Malmesburys Erwähnung einer cantilena Rollandi haben wird, und erklärt es schon bei Alpert mit «c'està-dire des chansons de geste» (1960: 382). Er ist offenbar durch Gaston Paris', Rajnas und Bédiers Kontroversen über «Kantilenen` als Vorstufen der altfranzösischen Epen so geprägt, dass er an keine andere Bedeutung denkt. Der ganze Zusammenhang seines Buches zeigt, dass er damit glaubt, in Bédiers «silence des siècles», jene massivste Verteidigungsbastion der Individualisten, vom zeitlichem Ende dieses Schweigens aus die bisher größte Bresche geschlagen zu haben - zeitlich viel einschneidender, als es die Nota Emilianense (1960: 384447) oder das bei Hastings gesungene Rolandslied (op. cit. 271) waren, besser datierbar, als es 1959 die Kommentare der Schreiber in einzelnen Einhart-Handschriften (op. cit. 282 f.) sein konnten, ebenfalls besser datierbar schließlich und von eindeutigerem Zeugniswert für den «Sitz im Leben〉 jener frühen volkssprachlichen Epik, als es die Olivier- bzw. Olivier-Roland-Namenmode (op. cit. 347-365) oder das Haager Fragment (op. cit. 372-381) sein konnten. Und um sozusagen das Eisen zu schmieden, solange es heiß ist, fügt er an die Präsenta-

8 Weit größere Wirkung hatte (und wie allgemein in der Forschung wird auch von mir im Folgenden zitiert) die schon ein Jahr später erschienene französische Fassung La Chanson de Roland et la tradition épique des Francs. Zur Alpert-Stelle dort ausfürhrlich p. $381 \mathrm{f}$., aber auch 81 und 393.

9 Von allen in den jährlichen Bibliographien der Société Rencesvals verzeichneten Rezensionen der beiden Menéndez-Pidalschen Bücher geht anscheinend nur die von Favati wenigstens in einem halben Satz (1960: 97) auf Alpert ein: „E resta il fatto che per trovare una citazione concreta di Rolando bisogna scendere nel tempo di almeno un altro secolo, e giungere al minimo al 999-1031, se è proprio di quella data (ma l'Aebischer ne dubita) il documento di Saint-Julien de Brioude che contiene la coppia onomastica Rolando-Olivieri, se non addiritura al 1020 circa, data alla quale Alperto di Metz, nella dedica del suo De diversitate temporum, cita una Cantilena Rollandi de W. Malmesbury.“ Ich kann nur hoffen, dass Favati hier einen Zeilensprungfehler seines Setzers übersehen hat! 
tion der Alpert-Stelle sogleich (op. cit. 382) den Schluss an, dass sich damit zwanglos aus solchen cantilenae auch die anderen relativ frühen Zeugnisse einer Karl-in-Spanien-Legende, speziell der enorme Tribut Saragossas, die Einnahme Córdobas und das Sonnenwunder, erklärten.

Auch Lecoy bemüht sich intensiv um den Sinn der Stelle. Schon 1959 (1960: 114) bezeichnet er die Entdeckung als «très importante», schränkt aber ein: «s'il [scil. Albert] parle en effet de cantilènes, il ne fait pas forcément allusion à des chansons de geste. Mais il est légitime d'en discuter.» Und 1963 (p. 121 f.) erkennt er im Gegensatz zu Siciliano und Horrent die Vergleichsstruktur der Stelle ohne Umschweife an: «Naturellement, pour que la phrase d'Albert de Metz ait un sens, il faut que les nouvelles «cantilènes〉 traitent de sujets analogues ou identiques aux anciennes» - wobei ich allerdings statt «sujets» ein engeres «sujets narratifs» und statt «analogues ou identiques» ein engeres "plus ou moins identiques» vorziehen würde. Doch beanstandet Lecoy - neben einem weiteren Detail von Menéndez Pidals Übersetzung - wieder energisch dessen Gleichsetzung von Alperts cantilenae mit chansons de geste. Nun ist sofort zuzugeben, dass cantilena schon in der Spätantike, dann auch im Mittelalter eine solche Fülle von Bedeutungsschattierungen aufweist, dass sich, anders als Menéndez Pidal implizit voraussetzt, vom Lexikon her dem Problem nicht beikommen lässt; vgl. die Artikel cantilena im TLL und inzwischen im MLW und LMLMAe. ${ }^{10}$ Die Diskussion muss sich vielmehr um die für etwa 1020 historisch nachweisbaren oder vermutbaren Fälle einer habituellen narrativen Fast-Identität von Älterem und Jüngerem drehen. In diesem Sinne fragt Lecoy denn auch: «[...] est-ce là le cas des «chansons de geste` seulement? N'en est-il pas de même pour toute forme de littérature populaire, et, en particulier, pour la chanson plus ou moins savante, dont on sait bien que les thèmes ne sont jamais très variés, sans parler de la lyrique religieuse?» Das klingt recht vage; man bekommt den Eindruck, dass Lecoy in defensiver Taktik die weniger ergiebigen Möglichkeiten aufsummieren will, um wenigstens deren kumulierte Wahrscheinlichkeit aufbieten zu können gegen die eine groß im Raume stehende Möglichkeit, dass es sich eben doch schon (zumindest: auch) um die Frühstufe der später manifesten - und dann für die jongleurs quantitativ weit ergiebigeren - volkssprachlichen Epik handelt. Doch wir brauchen zwischen Menéndez Pidals und Lecoys Auffassung nicht genauer abzuwägen. Denn einen viel gravierenderen Zweifel als denjenigen an der Gattung der cantilenae hat Lecoy abgedrängt in eine Fußnote (1963: 122 Anm. 2), wohl weil der vielbeschäftigte Herausgeber der Romania keine

10 Erst nach 1200 lässt sich das Wort vielleicht bei Johannes de Garlandia und Franco von Köln, sicher dann kurz vor oder um 1300 bei Johannes de Groche(i)o als terminus technicus für ,Sololied mit Chorrefrain“ nachweisen; vgl. hierzu auch Rohloff 1972: Register s.v. 
Muße fand, ihn auszuarbeiten. «Et je ne parle pas du fait qu'Albert de Metz, en dépit de son nom, écrit en terre germanique, pour des personnalités germaniques [...].» Soviel ich sehe, ist dieses Problem innerhalb der Romanistik nie weiterdiskutiert worden, obwohl nicht weniger auf dem Spiel steht als Alperts Frankophonie. Wie steht es um die?

Beginnen wir mit dem Empfänger von Alperts Widmung. Es ist Burchard von Worms, nach seiner Vita (ed. Waitz, 1841: 832) gebürtiger Hesse, nach heutiger genealogischer Erkenntnis verwandt mit „führenden Grafen- und Adelsgeschlechter[n] des nördlichen Hessen“ speziell um die obere Eder (LM s. v. 13 Burchard, nach W. Metz). Zwar wurde laut Sigeberts Gesta abbatum Gemblacensium (ed. Pertz 1841: 536) der Mönch, spätere Abt Olbert, den Sigebert noch persönlich kennen lernte, aus dem romanischen Gembloux zu Bischof Burchard nach Worms geschickt, um ihn bei der Ausarbeitung des Decretum zu unterstützen. Doch die Behauptung der etwa zwei Generationen jüngeren Gesta abbatium Lobbiensium («continuata〉, ed. Arndt 1869: 309 f.), Burchard sei damals vielmehr Kanonikus in Lüttich gewesen, wird heute durchweg abgelehnt, und dass Burchard zum Studium in Lobbes gewesen sei, glaubt überhaupt erst Trithemius kombinieren zu dürfen. ${ }^{11}$ Doch wie dem auch sei, jedenfalls ist Burchard deutscher Muttersprachler. Und da er, Alperts «tuo consilio» zufolge, den Kantilenen-Vergleich überhaupt angeregt hat (oder, wenn das eine bloße Höflichkeitsformel sein sollte, ihn nach Alperts Einschätzung zumindest voll billigt), gerät Alperts Aussage bereits in ein merkwürdiges Zwielicht. Wenn ein deutscher Muttersprachler - oder zumindest: wenn man zu einem solchen - von Kantilenen redet, ohne deren Sprache anzugeben, wieso ist da eher von französischen die Rede als von deutschen, deren Existenz um diese Zeit außer Zweifel stehen dürfte ${ }^{12}$

Betrachten wir weiter, was sich von Alperts eigenem Lebenslauf eruieren lässt! Die Stadt Metz und das umgebende pays messin scheinen seit antiken Zeiten immer romanophon geblieben zu sein. Darauf baut offensichtlich Menéndez Pidal, wenn er «Albert de Metz» mit Selbstverständlichkeit als Romanen betrachtet. Nun wäre es wohl hyperkritisch, zu bezweifeln, dass Alpert irgendwann in seiner ersten Lebenshälfte Mönch in Metz war. Sein Bericht über die

$11 \mathrm{Zu}$ diesem Fragenkomplex vgl. Bubenheimer 1972 passim, speziell 322 Anm. 13.

12 Zwischen einerseits Karls des Großen verlorener Sammlung heidnischer Heldenlieder, der Niederschrift des Hildebrandsliedes und dem Nibelungenstoff, wie ihn z. B. der Walthariusdichter kannte, andererseits etwa der Vorliebe des Bischofs Gunther von Bamberg († 1065) für Spielmannsdichtung und Dietrichsepik mag die latente Stoffgeschichte schwer zu verfolgen sein, und die cantilenae mag man sich sehr verschieden lang vorstellen - doch von einer Diskontinuität kann hier nicht die Rede sein. 
letzten Lebensjahre Bischof Dietrichs I. († 984) ist - ungleich einer Vita - nicht zum Lobe des Bischofs, sondern zur Entschuldigung der Tatsache geschrieben, dass Dietrich nach dem plötzlichen Tod Ottos II. (983) nicht dessen schon gekrönten 3-jährigen Sohn Otto III. unter der Vormundschaft seiner Mutter Theophanu, sondern aus Abneigung gegen die politische Macht einer Frau und noch dazu einer Griechin ${ }^{13}$ einige Monate lang Ottos II. Vetter Herzog Heinrich von Bayern als Kronprätendenten unterstützt hatte. Alpert stützt sich auf Aussagen eines Anonymus aus dem engsten Umkreis des Bischofs, nennt Dietrich aber auch im eigenen Namen noster presul und widmet seinen Bericht dem Abt Konstantin des Metzer Klosters St. Symphorien. ${ }^{14}$ Beide Werke Alperts sind nur in einer, nämlich derselben, Miszellanhandschrift des späten 11. Jahrhunderts auf uns gekommen; sie stammt, auch auf Grund ihrer sonstigen Inhalte, so gut wie sicher aus Metz und sehr wahrscheinlich aus dem Kloster St. Vincent, das Dietrich gegründet hatte und das Alpert ausführlich erwähnt. ${ }^{15}$ Schon der Bericht über Dietrich und viel stärker noch De diversitate sind gespickt mit sprachlichen Anklängen an Cäsars Bellum Gallicum, ${ }^{16}$ und St. Vincent besaß im 11. Jahrhundert eine Cäsar-Handschrift ${ }^{17}$ - beides damals noch recht atypisch. Vor allem aber: 67 Jahre nach Dietrichs Tod begann ein so umsichtiger Historiker wie Sigebert von Gembloux seine 20-jährige Tätigkeit in eben diesem Metzer Kloster; wenn er, der nach Ausweis seiner Schriften als einziger mittelalterlicher Autor beide Schriften Alperts benutzte, ${ }^{18}$ Alpert einen monachus Mettensis nennt, ${ }^{19}$ wird er gewusst haben, wovon er sprach. Ausschließlich auf Sigeberts Nennung geht dann Alperts Beiname «von Metz〉 bei allen modernen Historikern zurück.

Doch ein völlig anderes Bild bietet später De diversitate temporum. Von «Metz in Belgien` hören wir nur, dass Heinrich II. es zwischen 1009 und 1012 zweimal belagerte, dabei teilweise zerstörte und schließlich unterwarf, wobei Alperts Sympathie jetzt klar auf Seiten des Kaisers ist. ${ }^{20}$ Thema des Werkes auf seinen heute mehr als 50 Druckseiten sind vielmehr in erstaunlicher Detailfülle

13 Van Rij 1980: 110 und 114. Gute Charakterisierung von Alperts Darstellung bei Hugenholtz 1966: $257 \mathrm{f}$.

14 Van Rij 1980: 116 und 120.

15 Vgl. oben Anm. 5 und van Rij 1980: 116, 118..

16 Nach Manitius' Urteil (1888: 203), der dem Phänomen einen Aufsatz widmete und über 80 Stellen aushob, wird Alpert in der sprachlichen Benutzung Cäsars wohl von keinem anderen Schriftsteller des Mittelalters übertroffen. Van Rij 1980: XXXIII-XXXVI und 123-129 hat die Zahl der Nachweise sogar auf 190 erhöhen können.

17 Van Rij 1980: XIII.

18 Van Rij 1980: XXXIIf.

19 Vgl. oben Anm. 1.

20 Van Rij 1980: XI und 16. 
drei Jahrzehnte (etwa 990 bis 1021) der Geschichte eines schmalen Gebietsstreifens längs des Niederrheins bzw. seiner Arme von Elten bis Tiel und Utrecht. Alpert baut eine eigene kleine Vita des Bischofs Ansfried von Utrecht (sedit 990-1005) ein, worin er z. B. eine Örtlichkeit sechs Meilen außerhalb der Stadt sehr genau beschreiben kann. ${ }^{21}$ Ein Utrechtsches Heer im Kampf gegen Eindringlinge aus der Grafschaft Holland sowie die Verteidiger von Tiel gegen eine normannische Bedrohung nennt er nostri, ferner den Autor eines Lobgedichtes auf den Utrechter Bischof quidam e nostris, offensichtlich einen seiner Mitkleriker. ${ }^{22}$ Über die Unsitten der Kaufleute von Tiel - von ihren ständigen Klagen und ihrem unmoralischen Handelsrecht über ihre Neigung zu Meineid und Ehebruch bis hin zu morgendlichen Trinkgelagen auf gemeinsame Kosten und mit schmutzigen Scherzreden - verbreitet er sich ohne Not so detailliert, dass Historiker danach umfangreiche Aufsätze über die 〈Tieler Kaufmannsgilde um 1000〉 schreiben konnten. ${ }^{23}$ Die Pfalz zu Nijmegen ist für ihn kurz «die Pfalz〉. ${ }^{24}$ Doch hauptsächlich handelt er von der alten, als Verwaltungseinheit gerade aus der Geschichte verschwindenden Grafschaft Hamaland ${ }^{25}$ mit Elten als Schwerpunkt der Handlung. Hier geht es um den Untergang der Hamaländer Grafenfamilie: Graf Wichmann hatte das Kloster Elten gestiftet, ihm den Großteil seines Besitzes vermacht und seine Tochter Liutgard als Äbtissin eingesetzt; deren Schwester Adela ficht nun nach seinem Tod die Stiftung an, heiratet zur Erreichung ihrer Ziele einen unebenbürtigen Gewaltmenschen und stiftet ihn im Laufe einer wüsten Fehde zum Mord an. Adelas Charakterisierung durch Alpert ist verblüffend individuell und kaum ohne persönliche Bekanntschaft denkbar: Sie spricht zu laut, benutzt laszive Ausdrücke, hat einen unsteten Augenaufschlag, bringt aber ihren Dienerinnen ausgezeichnete Webetechniken bei und übertrifft im Verfertigen kostbarer Kleider fast alle Frauen «unserer Gegenden`. ${ }^{26}$

Angesichts eines solchen Befundes sind sich die Historiker einig: Alpert hat zumindest einen langen letzten Lebensabschnitt in dem genannten Gebiet verbracht. ${ }^{27}$ Dabei kommt ihnen noch ein glücklicher Zufall zu Hilfe. Alpert beginnt sein Widmungsschreiben an Burchard mit dem Satz: «Frater Immo causa

21 Van Rij 1980: 30.

22 Van Rij 1980: XV, XVII, 20, 26, 82.

23 Van Rij 1980: XV und 80. - Akkerman 1962 passim, Oexle 1989 passim.

24 Van Rij 1980: XV und 12.

25 Sie erstreckte sich von südlich Elten bis nördlich Deventer. Vgl.. Wirtz 1951 passim, speziell 50-80 mit Karte p. 55; van Winter 1980 passim.

26 Van Rij 1980: 10; Hugenholtz 1966: 255 f. Damit sei nicht gesagt, dass die Adela-Darstellung Alperts und auf ihm aufbauender Historiker objektiv sei; vgl. hierzu etwa Oediger 1954: 67-82, und Le Jan 2006 passim; weitere Lit. bei van Rij 1999: 40.

27 Van Rij 1980: XIVf. 
amoris ad me venit.» Das heißt im Zusammenhang: Aus der unmittelbaren Umgebung Bischof Burchards in Worms ist soeben ein gewisser Diakon Immo (den 1036 Konrad II. zum Bischof von Arezzo machen wird) auf einen Freundschaftsbesuch zu Alpert gekommen. Nun wissen wir aus den 1022 oder ganz kurz danach geschriebenen Miracula Waldburgae Tielensia, dass Immo gerade zu dieser Zeit Tiel besuchte und von dort den Text der Miracula nach Worms mitnahm; nichts liegt dann näher, als mit van Rij die Besuche Immos bei Alpert und in Tiel zu identifizieren, ${ }^{28}$ zumal es starke Indizien gibt, dass Alpertus der anonyme Autor der Miracula ist. ${ }^{29}$ Selbst wenn wir für Alpert weiterhin romanische Herkunft voraussetzen, spricht hier ein durch lange Jahre in germanophoner Umgebung gleichsam «naturalisierter» Autor zu einem Bischof deutscher Muttersprache von cantilenae schlechthin. Ist da nicht die Wahrscheinlichkeit, dass er romanische cantilenae meint, schon fast gleich Null?

Das ist noch nicht alles. Zwischen den beiden Teilen von Alperts Leben besteht eine unerwartete Verbindung. Sigebert von Gembloux hat in seinen Metzer Jahren auch die erste volle Vita Bischof Dietrichs verfasst; danach stammt dieser saus dem Gau Hamaland als Sohn des Grafen Eberhard >. ${ }^{30}$ Das ist anscheinend insofern nicht ganz exakt, als Eberhard nur als Graf des Sallandes, der Nachbargrafschaft des Hamalandes - und gleichzeitig der schon genannte Wichmann als Graf des Hamalandes - nachzuweisen ist. Doch waren beide verwandt, am ehesten Söhne von Brüdern. ${ }^{31}$ Fasst man also den Begriff Familie nicht ganz eng, so handelt Alpert in seinen beiden Werken essentiell von derselben niederländischen Familie!

28 Van Rij 1980: IX f. mit Anm. 8, XIV f.

29 Van Rij 1980 war in diesem Punkte noch misstrauisch, doch 1984 passim begründete er eindrucksvoll die Verdachtsmomente für die Identifizierung (und hielt sie 1999: speziell $17 \mathrm{f}$. und 32-35 aufrecht). War St. Waldburga damals allerdings Kanonikerstift - so zwei erzählende Quellen des 15. Jh. -, so müsste der frühere Mönch, wohl bei seiner Rückkehr in die Heimat, stattdessen die (geistliche, aber relativ bescheidene) Stelle eines custos bei den Kanonikern angenommen haben. Dazu passt, dass Alpert sich gegen Ende des Widmungsbriefes an Burchard (van Rij, 1980: 4, Z. 17 f.) scheinbar unmotivert als nicht ad opus Dei [...] idoneus bezeichnet (van Rijs Übersetzung: ,niet geschikt voor het koorgebed ‘ - und das Chorgebet ist die wichtigste raison d'être des abendländischen Möchtums!) und dass ihn der Bischof in seiner Antwort nur als specialis suus ,zijn dierbare vriend“ bezeichnet, also auffälligerweise darauf verzichtet, seine geistliche Stellung irgendwie zu präzisieren.

30 Sigebert, Vita Deoderici., ed. Waitz 1841: 464: „Deodericum, ex pago [...] Hamalant oriundum, comite Everardo patre“ [...].

31 So nach dem genealogischen System von van Winter 1980: 18 (mit Anm. 20), 25 und speziell 33; die essentielle Zusammengehörigkeit beider Teile der Familie und die sozusagen angestammten Rechte der Familie auf die Grafschaften Hamaland, Salland und Drenthe hatte schon Wirtz (1971: 46 f.) herausgearbeitet. 
Damit steht man vor einer klaren Alternative. Entweder ist ein frankophoner Alpert nach dem Tode seines Bischofs in dessen ferne Heimat übergesiedelt, ohne dass man dafür ein Motiv erkennen kann; das ist von einem einzelnen Historiker (Moll 1867: 344) angenommen worden. Nach der Vermutung aller anderen ist ein niederländischer Alpert aus seiner Heimat, zu Studienzwecken und/oder dem Bischof Dietrich nachziehend bzw. von ihm nachgezogen, nach Metz gekommen, doch einige Zeit nach dem Tode des verehrten Bischofs in seine Heimat zurückgekehrt. ${ }^{32}$

Die Versuchung liegt nahe, sich bezüglich der Herkunft Alperts eines letzten, scheinbar durchschlagenden Arguments $\mathrm{zu}$ bedienen. Wie oben zitiert, nennt Alpert den Wormser Diakon aus der Umgebung Bischof Burchards und späteren Bischof von Arezzo, also einen Weltgeistlichen, «frater Immo». Weil das Wort hier nicht〈Mönchsbruder, Frater〉 bedeutet, haben Pertz, Manitius, Pijnacker Hordijk und Wattenbach-Holtzmann-Schmale ${ }^{33}$ es als «leiblicher Bruder genommen. Nun hatte Immo einen bei Tiel verheirateten, also nicht mit Alpert identischen Bruder. ${ }^{34}$ Die Annahme, samt Alpert wären auch dieser und Immo aus der Romania gekommen, würde alle Wahrscheinlichkeit strapazieren. Vielmehr schiene die Situation der Familie im alternativen Sinne klar: Ein Bruder blieb in der niederländischen Heimat und trat vermutlich in die Fußstapfen des Vaters, die beiden anderen wurden Geistliche, vielleicht mit der Hoffnung, in der Umgebung von machtvollen Mitgliedern des Reichsepiskopats Karriere

32 Zu dieser Alternative van Rij 1980: XX. - Dietrich war Vetter Kaiser Ottos des Großen und damit auch seines Bruders Brun, Erzbischofs von Köln und Platzhalters des Kaisers für (Niederund Ober-)Loth(a)ring(i)en. Nach einem mindestens neunjährigen Aufenthalt bei Brun wird er von Otto und Brun als Bischof von Metz eingesetzt, vgl. z. B. Parisse 1965: 110-112. Nun dürften die Mitglieder des Ottonischen Reichsepiskopates noch ungenierter als die Bischöfe anderer Zeiten Landsleute in ihre Umgebung nachgezogen haben; so wissen wir von Dietrich, dass er seinen noch jungen Neffen Eberhard zur Ausbildung nach Metz geholt hatte (Sigebert, Vita Deoderici, cap. 19, p. 479 f.). Man kann sich leicht vorstellen, dass solche Landsleute sich nach dem Tod ihres Gönners isoliert fühlten und in die Heimat zurückkehrten. Ein mögliches Indiz für Dietrichs Anhänglichkeit an seine angestammte germanische Muttersprache liegt übrigens darin, dass Alpert und Sigebert ihn «Deodericus» nennen, nicht «Theodericus» mit lautgesetzlichem $/ \mathrm{t} /<$ germ. $/ \mathrm{p} /$, wie sonst der Name in der Romania immer lautet (frz. Thierry). Es muss das deutsche und niederländische $/ \mathrm{d} /</ \mathrm{d} /</ \mathrm{p} /$ vorliegen (dt. Dietrich, niederld. Diederik $>$ Dirk), eine Entwicklung, deren Ende man im Rheinfränkischen auf (kurz) «nach 900», im Mittelfränkischen auf das «10. und 11. Jahrhundert», im Niederländischen immerhin auf eine Zeit «vóór 1100» zu datieren pflegt (Braune-Eggers § 167, van Loey §50). Der Bischof selbst muss auf dieser Namensform gegen die allgemeine Kanzleipraxis bestanden haben, da sonst der äußerst belesene Romane Sigebert sie nicht übernehmen würde.

33 Die Stellenangaben bei van Rij 1980: IX Anm. 4.

34 Auch das erfahren wir aus den Miracula Waldburgae Tielensia, vgl. van Rij 1980: X, und 1984: 84. 
zu machen. Aber gegen diese Argumentation erhebt van Rij (1980: Xf.) Einspruch: im Text stehe nicht frater meus, und frater allein könne - so van Rij dem Sinne nach - ein vages «Bruder in Christo» sein. Obwohl man hier durchaus geteilter Meinung sein kann, ${ }^{35}$ wollen wir auf das Bruder-Argument verzichten; die anderen Argumente sollten ausreichen, Alperts Namen definitiv aus der (Vor-) Geschichte der altfranzösischen Literatur zu streichen.

Nun zum Pseudo-Alkuin! Schon Gaston Paris (1865/2 1905: 492) machte aufmerksam auf das Ms. Paris, Bibliothèque Nationale. lat. 5354, das er nur grob auf das 11. Jahrhundert datierte; der bekannte Historiker Robert Fawtier (1933: 81) engte dann paläographisch die Datierung ein auf «um 1050, eher früher als später>. Diese Handschrift enthält unter vielem anderen ${ }^{36}$ Einharts Karlsvita mit Nennung des Verfassers («Vita [..] gloriosissimi imperatoris Karoli [...] aedita ab Aeginardo»). ${ }^{37}$ Doch am Ende des Textes fügt der Schreiber hinzu: «Reliqua actuum eius gesta / seu et que in carminibus vulgo canuntur de eo / non hic pleniter sunt descripta / sed require in vita quam alchuinus de eo scribit.» ${ }^{38}$ Gaston Paris zweifelte an der Existenz der hier erwähnten Karlsvita des (Pseudo-) Alkuin, weil man sonst nichts von ihr wisse. Doch Menéndez Pidal, der gleich nach der Besprechung Alperts auf die Handschrift zu sprechen kommt (1960: 382f. mit Anm. 2) und Fawtiers Datierung akzeptiert, fragt: «Comment expliquer la citation d'une œuvre inexistante?» Er vermutet ein Werk in der Art des etwa ein Jahrhundert jüngeren Pseudo-Turpin, doch «moins arbitraire [...] et plus fidèle au récit des «chansons»».

Leider fällt es auch hier schwer, Menéndez Pidal zuzustimmen, und zwar auf Grund von Tatsachen, die erst dank der monumentalen Studie von Matthias Tischler (2001) zur Überlieferungsgeschichte der Einhartschen Karlsvita klar vor uns liegen. ${ }^{39}$ Es gibt nämlich unter deren Handschriften eine Reihe, in denen

35 Denn wenn Immo nicht Alperts Bruder ist, zwischen beiden also nicht die als natürlich vorauszusetzende Bruderliebe waltet, ist das knappe «causa amoris« (nicht «causa amicitiae»!) merkwürdig. Jedenfalls erhält man aus dem Brief des custos (vermutlich also Alperts, vgl. oben Anm. 29) an Immo (van Rij 1984: 88 f.) trotz der stilistischen und motivischen Anlehnung an Sulpicius Severus (van Rij 1984: 89-91) den Eindruck, dass zwischen beiden seit langem eine enge Vertrautheit besteht, wobei der custos der Ältere ist - sonst könnte er auch im Scherz nicht zu sagen wagen, der ihm jetzt (aber anscheinend erst seit kurzem, «nunc») sozial Überlegene hätte Kerker, Fesseln und Block verdient, weil er ohne Vorwissen des «custos» dessen «conclavia» und «cellula» durchsucht hatte.

36 Vgl. Tischler 2001: 1142-1146.

37 Vgl. den umfangreichen vollen Titel bei Tischler 2001: 912 Anm. 33.

38 So Tischler 2001: 487 Anm. 803. Menéndez Pidal hat «ea quae» statt «et que».

39 Tischlers Studie, LXX + 1828 [sic] Seiten, ist eine während mehrerer Postgraduiertenjahre und dank ausgedehnter „Handschriftenreisen durch Europa“ (Vorwort, p. V) erweiterte Heidelberger Dissertation. Ich gestehe, dass ich mir eine so intensive, minutiöse und erfolgreiche 
das Werk fälschlich Alkuin zugeschrieben wird (Tischler 2001: $486 \mathrm{mit}$ Anm. 803, vgl. auch 23, 28, 35, 37, 40):

- Paris B. N. lat. 6186, zweite Hälfte des 13. Jahrhunderts, im 17. Jahrhundert bei den Karmelitern von Clermont-Ferrand;

- Paris B. N. nouv. acq. lat. 2664, geschrieben etwa im ersten Viertel des 11. Jahrhunderts in Cluny;

- Leiden B.P.L. 20, geschrieben zwischen 1139 und 1149 in Le Bec; hier hat ihr wohl erster Besitzer, der illustre Robert de Torigny, den Genitiv alcvini eigenhändig zugesetzt;

- Cambridge Gonville and Caius College 177/220, geschrieben bald nach der Mitte des 12. Jahrhunderts in Reading, eine Abschrift der vorigen mitsamt Alkuins Namen; schließlich

- Vatikan Pal. Lat. 1569, geschrieben Ende des 15. Jahrhunderts in Venedig.

Ferner kannten im anglonormannischen Herrschaftsgebiet außer Robert de Torigny auch Radulfus de Diceto ( $†$ um 1202) und Giraldus Cambrensis († 1223), im kapetingischen Herrschaftsbereich Alberich von Troisfontaines ( 1252 oder kurz darauf), in Italien Jacobus a Voragine († 1298) und Petrarca (laut Brief von 1365) eine «Karlsvita von Alkuin», wobei es sich nach Tischlers Nachweisen (mit uneigentlicher, durch die Kürze der Erwähnung sattsam erklärter Ausnahme des Radulfus de Diceto) immer eindeutig um Einharts, nicht um ein anderes, Werk handelt.

Insgesamt stammt diese Zuschreibungstradition zweifellos aus Zentralfrankreich. Ihre älteste erhaltene Handschrift aus dem Cluny des frühen 11. Jh. hängt stemmatisch an einer Einhart- (und Astronomus-, also «Kaiserdiptychon»-) Tradition des 9. Jh. aus Orléans, die noch keine Zuschreibung an Alkuin kennt (Tischler 2001: 1102-1118, vgl. 1142). ${ }^{40}$ Nun wussten im Westreich anders

Erforschung der Einhartschen Überlieferungsgeschichte (vom Auffinden von Einhart-Handschriften über die Handschriften- und Editionsgeschichte selbst des 16. bis 18. Jh. bis zur Beherrschung eines enormen Maßes an Sekundärliteratur) vorher nicht einmal als Desiderat der Forschung habe vorstellen können. Auch die Romanistik kann aus dem Werk noch mehrfachen Nutzen ziehen.

40 Im 19. Jahrhundert behaupteten zwei Lokalhistoriker des Klosters Cluny bzw. seiner einstigen Bücherschätze, die diese Handschrift «von Alkuins Karlsvita» für verschollen hielten (während sie nachweislich vom 18. Jh. bis 1901 an wenig bekannter Stelle in Lyon lagerte, Tischler 2001: 37), sie sei schon von Karl dem Einfältigen dem Herzog Wilhelm von Aquitanien und von diesem dem ersten Abt von Cluny, Berno, geschenkt worden, somit «le plus ancien [scil. manuscrit] de l'abbaye» gewesen (Tischler, op. cit. 910 f. mit Anm. 31). Das kann zwar für das erhaltene Exemplar aus paläographischen Gründen so nicht zutreffen, aber doch in das Entstehungsmilieu der Alkuin-Zuschreibung zurückweisen. Tischler (2001, 913) erinnert in diesem Zusammenhang daran, dass der erste große Abt des noch jungen Cluny, Odo (927-942), in Saint-Martin de Tours erzogen und von dort nach dem Zeugnis seines Schülers und ersten Biographen über Baume-les-Messieurs «hundert Handschriften» nach Cluny mitbrachte. 
als im deutschen Sprachgebiet viele fast nichts von Einhart, umso mehr von Alkuin. Er war als Haupt von Karls Hofschule, als Abt von Saint-Martin de Tours, dem ehrwürdigsten Kloster des Gesamt-, dann des Westreiches, als ungemein fruchtbarer Autor und nicht zuletzt durch die im westfränkischen Ferrières entstandene Vita Alcuini in Erinnerung, ohne dass deshalb jedermann auf den Gedanken kommen musste, Alkuins Todesjahr 804 mit Karls Todesjahr 814 zu vergleichen. Wer wenn nicht Alkuin war dann berufen gewesen, Karls Historiograph zu sein - so muss sich ein westfränkischer Abschreiber gefragt haben, der die Brillanz des Lateins und (vor allem aus der praefatio) die Nähe des Autors zu Karl erkannte. Tischler (2001: 912, vgl. 910) hält hier zudem eine anonymes Exemplar als Vorlage für möglich. Von Zentralfrankreich aus hat die Tradition dann unter ähnlichen Wissensvoraussetzungen den anglonormannischen Herrschaftsbereich und zögernder Italien erreichen können, marginal auch den frankophonen Nordosten, gar nicht das deutsche Sprachgebiet.

Durch diese Tradition der Fehlzuschreibung ändert sich die Struktur des uns beschäftigenden Problems beträchtlich. Wir interessieren uns für die Dinge, die bei Einhart nicht pleniter sunt descripta, vielmehr in carminibus vulgo canuntur, und konnten unerwartet der Aufforderung nachkommen require in vita quam Alchvinus de eo scribit - aber da steht nichts dergleichen! Will man jetzt noch Menéndez Pidal folgen, so ist man gleich mit zwei «Karlsviten von PseudoAlkuin» konfrontiert: der gut bezeugten realiter einhartischen und einer nicht erhaltenen, nur in einer Handschrift erwähnten epikfreundlichen. Hier kann die Gleichheit der Zuschreibung doch kaum Zufall sein. Wie erklärt sie sich dann? Wie hängen die beiden zusammen? War die epikfreundliche mit der anderen identisch (also in Wirklichkeit einhartisch), nur angereichert durch Motive aus der frühen Epik? Das anzunehmen wäre unfundiert; denn noch bei keiner der 123 heute bekannten (vollständigen oder fragmentarischen) Vita-Karoli-Handschriften ${ }^{41}$ - geschweige denn bei einer vor 1100 entstandenen - hat man je ein Eindringen solcher Motive in den Vita-Text feststellen können; ${ }^{42}$ dafür waren eben einerseits der Respekt vor der offensichtlichen Karlsnähe und der Brillanz des Autors, andererseits das weit verbreitete Misstrauen gegenüber allem Jongleurwissen zu groß. Dann bleibt nur eines: Der Schreiber von Paris lat. 5354 hat sich partiell geirrt. Er hatte soeben die Karlsvita eines ihm bis dato unbekannten Aeginardus abgeschrieben und war enttäuscht; denn er vermisste dort Motive, die ihm aus Liedern vertraut waren und die wenigstens er - das implizieren seine Worte ja eindeutig - für glaubhaft hielt. Aus Gesprächen mit anderen

41 Vgl. die Liste bei Tischler, 2001: 20-44.

42 Ich spreche nicht von einer vereinzelten Randbemerkung nach 1100 in der Handschrift London, British Library, addit. 21109 (Menéndez Pidal 1960: 383 mit Lit.). 
Mönchen, so dürfen wir annehmen, wusste er, dass es auch eine «Karlsvita von Alkuin» gab, von dem großen Alkuin, der doch Karl gewiss näher gestanden hatte als jener Aeginardus und der schon deshalb vollständiger berichtet haben musste als dieser. Bei Alkuin müsste man nachlesen .... Und schon floss dem Schreiber die teilweise falsche Bemerkung aus der Feder. Nur mit dieser Hypothese wird man meines Erachtens den scheinbaren Pseudo-Alkuin-Zwillingen gerecht, ohne den Zufall zu strapazieren. Streichen wir also besser - mit Gaston Paris - auch den Pseudo-Alkuin aus der Geschichte der altfranzösischen Epik!

Zur Vorgeschichte der altfranzösischen Epik haben wir somit zwei - wie mancher Forscher genüsslich sagen würde - mythes aufgeben müssen. Heißt das nun, dass die Neotraditionalisten Unrecht haben? Noch keineswegs. Es bleibt im Zeugnis des Schreibers von Paris lat. 5354 immerhin eine Aussage, bei der er sich nicht irren kann: dass nämlich schon zu seiner Zeit über Karl den Großen Dinge, die nicht bei Einhart zu finden sind (also auch für uns im Verdacht der Fiktivität stehen müssen), in Liedern (also vielleicht variablen, aber doch wiedererkennbaren Einheiten) volkssprachlich (denn vulgo im Volke kann sich nicht auf lateinische Lieder beziehen) gesungen (also nicht als gesprochene Anekdoten tradiert) wurden, und zwar in solchem Umfang, dass einfache Gemüter ihr Fehlen in einer Karlsbiographie mit Enttäuschung an dieser quittierten. Das ist weit mehr, als Alperts cantilenae bestreitbarer Gattung und ungenannter Thematik zu bieten hätten, selbst wenn sie romanisch wären!

Umso wichtiger wird es damit, die Handschrift mit den heutigen Mitteln im breiten Geflecht der Einhart-Überlieferung zeitlich-räumlich möglichst genau zu situieren. Von Fawtier (1933: 81) wurde sie, wie gesagt, paläographisch «um 1050, eher früher als später> datiert, mit etwas anderer Akzentuierung dann von Ernst Tremp (1991: 58 f.) auf «erste Hälfte / Mitte des 11. Jahrhunderts». Da sie einen Besitzvermerkung des Klosters Bonneval (Diözese Chartres) aus dem 13. Jh. enthält, hielt Tremp sie für «mit großer Wahrscheinlichkeit» dort auch geschrieben.

Sehr eingehend behandelt sie schließlich Tischler (2001); laut Register wird sie an 30 Stellen des Werkes erwähnt, passim (z. B. 2001: 808-816 und 11111118) werden aus ihr Lesungen zitiert, ein 14-seitiger, auf Autopsie beruhender Abschnitt ist ausschließlich dieser Handschrift gewidmet (2001: 1142-1156). Nach Tischler «dürfte [sie] bald nach der Jahrtausendwende entstanden sein, also früher, als bisher allgemein angenommen» (2001: 1142, ähnlich 34, 104), zwar frühestens zur Zeit des Abtes Rainard von Bonneval, d.h. zwischen 979 und 1015 (2001: 1155, vgl. 1153), aber noch im «1. Drittel des 11. Jahrhunderts» (2001: 487 Anm. 803), und zwar gleich für Bonneval im knapp 100 km entfernten Fleury. In diesem Zusammenhang kann Tischler nämlich zeigen (2001: 1115), dass schon im späteren 9. Jahrhundert, dem Stemma nach aus der Gegend von 
Orléans kommend, in Fleury eine Handschrift der Vita Karoli verfügbar war, welche Adrevald von Fleury für seine Miracula Sancti Benedicti benutzte; ferner, dass mit dieser Vorlage Adrevalds auch unsere Handschrift Paris lat. 5354 sowie die umfangreichen Einhart- (und Astronomus-)Auszüge, die Hugo von Fleury 1110 in die sechsbändige Fassung seiner Historia ecclesiastica einarbeitete, unstreitig eine Familie bilden, doch so, dass unsere Handschrift und Hugos Auszüge noch weit enger zusammenstimmen, ohne dass unsere Handschrift die Vorlage Hugos gewesen sein kann (2001: 1148-1152); unsere Handschrift und Hugos Exzerpte entstammen also (zumindest indirekt, wahrscheinlicher direkt) derselben, schon nach Fleury gehörenden Vorlage. Eines unter sehr vielen Beispielen für den letztgenannten Sachverhalt mag genügen: Unsere Handschrift und Hugo bieten an der Roland-Stelle in Einharts Kapitel 9 die Mitteilung, Eggihard, Anselm und Roland seien gefallen cum aliis quam pluribus «mit sehr vielen anderen $>^{43}$ statt Einharts cum aliis compluribus «mit nicht wenigen anderen (2001: $1148 \mathrm{f}$.) - im frühen 11. Jahrhundert eine auch für die Romanistik inhaltlich interessante Variante, die übrigens in keiner Vita-Karoli-Ausgabe des 19. oder 20. Jahrhunderts aufgeführt ist! Nun war Fleury ja nicht nur schon seit der hochkarolingischen Zeit ${ }^{44}$ allmählich $\mathrm{zu}$ einem Skriptorium allerersten Ranges geworden; ${ }^{45}$ seit etwa 950 war es auch ein wichtiger Ausgangspunkt der Reform von Klöstern. Insbesondere führte Fleury um 978 die Reform, ja faktisch die Wiederbegründung, des von den Normannen zerstörten Bonneval durch und schickte Mönche mit Rainard als Abt dorthin, der, wie gesagt, bis 1015 amtierte. Aus Fleury stammte in Bonneval auch noch Abt Tetfrid, dessen Streit mit den eigenen Mönchen 1023 durch Abt Gauzlin von Fleury und den zuständigen Bischof Fulbert von Chartres geschlichtet werden musste (2001: $1153 \mathrm{f}$. mit Anm. 776). Da ist es selbstverständlich, dass Fleury Bonneval gerade in dessen Anfängen unter Rainard und Tetfrid auch mit Handschriften versorgte - darunter eben unserer Handschrift, wobei wir es dahingestellt sein lassen dürfen, ob dazu in Fleury eigene Schreiber oder solche aus Bonneval tätig waren (zur Schreiberfrage vgl. Tischler 2001: $1146 \mathrm{f}$. und 1155). ${ }^{46}$

43 Complures ,nicht wenige“ ist klassisch. Quam plures (manchmal quamplures geschrieben) findet sich seit Plautus selten und meist unsicher belegt (TLL s. v. complures, col. 2107, Z. 19 ff., Forcellini s.v. quamplures ,molti assai'); etwa von Hieronymus an ist es häufiger, und zwar jetzt - wie das klassische quam plurimi - in der Bedeutung ,wie viele doch, sehr viele' (TLL s. v. multus, col. 1612, Z. 66 f., klarer Blaise s. v. quam 3: ,le plus possible', ,très').

44 Interessante Schlaglichter bei Bischoff 1979, vgl. das Register p. 337.

45 Vgl. etwa Pellegrin 1988: 159-210, 285-297, Mostert 1989 passim.

46 Fleury und Bonneval liegen beide innerhalb oder zumindest am Rande jenes sich von Cluny zum anglonormannischen Bereich hinziehenden Gebietes, wo man die «Karlsvita von Alkuin» zu kennen glaubte, und erfüllen damit auch im geographischen Sinne die Vorausset- 
Nun sind Romanisten ja oft gegenüber paläographischen Datierungen recht kritisch eingestellt. Das ist verständlich, wenn die geographische Heimat der Handschrift unbekannt ist, so dass die nur sehr allmähliche räumliche Ausbreitung von Neuerungen nicht adäquat berücksichtigt werden kann, oder auch, wenn die Datierung der Handschrift überhaupt erst um ihrer epengeschichtlichen Relevanz willen durchgeführt wird, so dass unvermerkt Vorerwartungen in sie einfließen können; beides trifft z. B. zu für das Haager Fragment oder in geographisch bescheidenerem Umfang - für die Oxforder Handschrift des Rolandsliedes. Im vorliegenden Falle aber ist jetzt die Heimat der Handschrift mit der wünschenswerten Genauigkeit bekannt, und niemand, der Tischlers Ausführungen nachliest, wird ihn verdächtigen, bei seiner Datierung beeinflusst worden zu sein durch die oben herausgearbeitete romanistische Relevanz des canuntur-Nebensatzes oder gar der quam-pluribus-Variante. Wollen wir trotzdem bewusst vorsichtig verfahren, so können wir ja um den Mittelpunkt der vorgeschlagenen Datierung «erstes Drittel des 11. Jahrhunderts», also um das Wahrscheinlichkeitsmaximum in den Jahren 1016/1017, noch den Sicherheitsradius von einer Generation, d. h. von etwa dreißig Jahren, legen.

Auch dann bleibt der canuntur-Nebensatz ein Zeugnis gerade noch aus der ersten Hälfte des 11. Jahrhunderts - und damit das älteste Zeugnis dafür, dass legendäre Karlsmotive in Liedern vorgetragen wurden, und die quam-pluribusVariante bleibt ein zumindest erwägenswertes Indiz für die Möglichkeit, dass dabei schon die Zahl der epischen Roncevaux-Opfer anzuschwellen begann.

Abschließend sei der Versuch gestattet, dieses Ergebnis mit wenigen andeutenden Strichen in einen größeren Zusammenhang zu stellen. Geographisch wie zeitlich steht das canuntur-Zeugnis zwischen den beiden meines Erachtens am ehesten resistenten Indizienkomplexen einer beginnenden Roland-Epik: nämlich zwischen einerseits der Olivier-Namenmode, die gegen 1000 nahe Vienne und damit - was bisher so gut wie unbeachtet blieb - nahe dem Ursprungspunkt der Gottesfriedens-Bewegung begonnen hatte, und andererseits jenen Indizien, die seit etwa dem letzten Drittel des 11. Jahrhunderts auf Westfrankreich (Anjou, Normandie) verweisen werden. Insgesamt wird man bei der Suche nach solchen Indizien - und auch allgemeiner nach potentiell epenträchtigen Namen - fündig nicht so sehr im eigentlichen Südfrankreich (und damit im Bereich einer vermeintlich okzitanischen Karlsepik), sondern entlang erst der südlichen, dann der westlichen Randzone des realen kapetingischen Machtbereichs, wohingegen dessen Hauptgebiet auffälligerweise erst nach 1100 ins Bild kommt. Diese 〈Verspätung〉 des kapetingischen Hauptgebiets dürfte ein-

zung unserer weiter oben gegebenen Inexistenz-Erklärung des epenfreundlichen PseudoAlkuin. 
fach - aber wiederum bisher unbeachtet - darin begründet sein, dass die Ablösung der Karolinger durch die Kapetinger zu den längsten und schmerzvollsten Ablösungsprozessen gehört, die die europäische Geschichte kennt. So etwas wirkt lange nach, so dass im unmittelbaren Kapetingerbereich lange auch breiten Bevölkerungsschichten eine einschränkungslose emotionale Identifizierung mit der Sache der Karolinger - wie eine Epik dieser Art sie braucht - inopportun erscheinen musste. Das Bestreben der Kapetinger, sich nach dem rechtlichen schließlich auch den ideologischen Mantel der Karolinger überzuwerfen, manifestierte sich einigermaßen klar erst, als sie sich spät im 11. Jahrhundert den Karolingernamen Ludwig aneigneten, und konnte sich voller erst entfalten, als seit 1122 Abt Suger von Saint-Denis sein geniales Gespür für das Potential von Symbolen in den Dienst des Königshauses und zugleich seiner eigenen Abtei stellte. Selbst nach diesem Datum wird ader epische Karl nur sehr allmählich seinen Hauptsitz von Aachen nach Paris verlegen und schließlich zum ,König von Saint-Denis‘ - und sein Reich zum ,Königreich von Saint-Denis‘ - werden.

\section{Zitierte Literatur}

Akkerman, J.B., 1962: «Het koopmansgilde van Tiel omstreeks het jaar 1000», in: Tijdschrift voor Rechtsgeschiedenis 30, 409-471.

Alpert von Metz: siehe die Editionen von Dederich, Eccard, Hulshof, Migne, Pertz, Pijnacker Hordijk und vor allem van Rij.

Arndt, Wilhelm, ed., 1869: «Gesta abbatum Lobbiensium», in: Monumenta Germaniae Historica, Scriptores, XXI, Hannover 1869, 307-333.

Bédier, Joseph, 1904-1908, 31926-1929: Les légendes épiques, 4 Bde., Paris, Champion. Bischoff, Bernard, 1979: Paläographie des römischen Altertums und des abendländischen Mittelalters, Berlin, Erich Schmidt.

Blaise, Albert, 1954: Dictionnaire latin-français des auteurs chrétiens, Turnhout, Brepols. Braune-Eggers, 1975: Wilhelm Braune, Althochdeutsche Grammatik, 13. Aufl. besorgt von Hans Eggers, Tübingen, Niemeyer, 1975.

Bubenheimer, Ulrich, 1972: «Der Aufenthalt Burchards von Worms im Kloster Lobbes als Erfindung des Johannes Trithemius», in: Zeitschrift der Savigny-Stiftung für Rechtsgeschichte, Kanonistische Abteilung, 58, 320-337.

Dederich, Andreas, ed. et trad. (dt.), 1859: Des Alpertus von Metz zwei Bücher über verschiedene Zeitereignisse, nebst zwei Bruchstücken über Bischöfe von Metz, Münster, Coppenrath.

Eccard, Johann Georg von, ed., 1723: «Alpertus Mettensis, De diversitate temporum, et: Fragmentum de Deoderico primo episcopo Mettensi», in: J.G.v.E., Corpus historicorum medii aevi, I, Leipzig, Gladitsch, col. 91-132.

Faral, Edmond, 1909, 21971: Les jongleurs en France au moyen âge, Paris, Champion. Favati, Guido, 1960: Rez. zu Menéndez Pidal 1959. In: Studi francesi 4, 95-99.

Fawtier, Robert, 1933: La Chanson de Roland: étude historique, Paris, de Boccard. 
Forcellini: Egidio Forcellini, 1965: Lexicon totius latinitatis, 4. Aufl., 2. Nachdruck, 6 Bde., Bologna, Forni.

Förstemann, Ernst Wilhelm, 21900: Altdeutsches Namenbuch, I, Personennamen, Bonn, Hanstein.

Gesta abbatum Lobbiensium: siehe die Edition Arndt.

Horrent, Jules, 1951: La Chanson de Roland dans les littératures française et espagnole au moyen âge, Paris, Belles Lettres.

Hugenholtz, F.W.N., 1966: «Alpertus Mettensis als ,biograaf"», in: Tijdschrift voor geschiedenis 79, 249-259.

Hulshof, Abraham, ed., 1916: Alperti Mettensis de diversitate temporum. Met inleiding van C[ornelius] Pijnacker Hordijk, Amsterdam, Müller.

Johannes de Groche(i)o: siehe die Edition Rohloff.

Kauffmann, Henning, 1968: Ergänzungsband zu Förstemann 1900, München, Finck, und Hildesheim, Olms.

Le Jan, Régine, 2006: «La vengeance d'Adèle ou la construction d'une légende noire», in: La vengeance, 400-1200, ed. Dominique Barthélemy [et al.], Rom, École française de Rome, 325-340.

Lecoy, Felix, 1960: [Diskussionsbeitrag vom 23. Juli 1959 auf dem Kongress der Société Rencesvals in Poitiers]. In: Bulletin bibliographique de la Société Rencesvals 2, 112-115.

Lecoy, Felix, 1963: Rez. zu Menéndez Pidal 1959 und 1960. In: Romania 84, 88-133.

LM: Lexikon des Mittelalters, I, München, Artemis u. a., 2003.

LMLMAe: Lexicon musicum latinum medii aevi, 1992-, ed. Michael Bernhard, München, Bayerische Akademie der Wissenschaften.

Manitius, Max, 1888: «Zu den Werken Alperts», in: Neues Archiv 13, 202-208.

Manitius, Max, 1923: Geschichte der lateinischen Literatur des Mittelalters, II, München, Beck.

Menéndez Pidal, Ramón, 1959: La Chanson de Roland y el neotradicionalismo, Madrid, Espasa Calpe.

Menéndez Pidal, Ramón, 1960: La Chanson de Roland et la tradition épique des Francs, deuxième édition, revue et mise à jour par l'auteur avec le concours de René Louis et traduite de l'espagnol par Irénée-Marcel Cluzel, Paris, Picard.

Mierlo, Jozef van, S.J., ed., 1935: Het Roelantslied, Gent, Erasmus.

Migne, Jacques Paul, ed., 1853: Patrologia Latina, CXL, Paris, Migne.

MLW: Mittellateinisches Wörterbuch, 1967-, begr. von Paul Lehmann und Johannes Stroux, München, Beck.

Moll, Willem, 1867: Kerkgeschiedenis van Nederland vóór de hervorming, II, 2, Utrecht, Kemink.

Mostert, Marco, 1989: The library of Fleury. A provisional list of manuscripts, Hilversum, Verloren.

Oediger, Friedrich Wilhelm, 1954: «Adelas Kampf um Elten (996-1002)», in: Annalen des Historischen Vereins für den Niederrhein 155/156, 57-86.

Oexle, Otto-Gerhard, 1989: «Die Kaufmannsgilde von Tiel», in: Untersuchungen zu Handel und Verkehr der vor- und frühgeschichtlichen Zeit in Mittel- und Nordeuropa, VI, Organisationsformen der Kaufmannsvereinigungen in der Spätantike und im frühen Mittelalter, ed. Herbert Jankuhn, Göttingen, Vandenhoeck und Rupprecht, 325-340.

Paris, Gaston, 1865, 21905: Histoire poétique de Charlemagne, Paris, Franck.

Parisse, Michel, 1965: «Thierry I ${ }^{\text {er }}$, évêque de Metz (965-984)», in: Les Cahiers lorrains N.S. $17,110-118$. 
Pellegrin, Élisabeth, 1988: Bibliothèques retrouvées. Manuscrits, bibliothèques et bibliophiles du moyen âge et de la Renaissance. Recueil d'études publiées de 1938 à 1985, Paris, Éditions du Centre National de la Recherche Scientifique.

Pellegrini, Silvio, 1964: Studi rolandiani e trobadorici, Bari, Adriatica Editrice.

Pertz, Georg Heinrich, ed., 1841: «Sigeberti Gemblacensis monachi Vita Deoderici primi episcopi Mettensis», in: Monumenta Germaniae Historica, Scriptores, IV, Hannover, Hahn, 461-483.

Pertz, Georg Heinrich, ed., 1841: «Alperti Mettensis monachi Fragmentum de Deoderico primo episcopo Mettensi [et] De diversitate temporum.» In: Monumenta Germaniae Historica, Scriptores, IV, Hannover, Hahn, 697-723.

Pertz, Georg Heinrich, ed., 1848: «Sigeberti Gemblacensis monachi Gesta abbatum Gemblacensium», in: Monumenta Germaniae Historica, Scriptores, VIII, Hannover, Hahn, 523-564.

Pijnacker Hordijk, Cornelius, ed. (Faks.), 1908: Alpertus Mettensis, De diversitate temporum und De Theoderico I episcopo Mettensi, Codex Hannoveranus 712A, in phototypischer Reproduktion. Einleitung von C. P. H., Leiden, Sijthoff.

Rajna, Pio, 1884: Le origini dell'epopea francese, Florenz, Sansoni.

Rohloff, Ernst, ed., 1972: Die Quellenhandschriften zum Musiktraktat des Johannes de Grocheio, Leipzig, Deutscher Verlag für Musik.

Schlaug, Wilhelm, 1955: Studien zu den altsächsischen Personennamen des 11. und 12. Jahrhunderts, Lund, Gleerup.

Schlaug, Wilhelm, 1962: Die altsächsischen Personennamen vor dem Jahre 1000, Lund, Gleerup.

Siciliano, Italo, 1951: Les origines des chansons de geste. Théories et discussions, traduit de l'italien par P. Antonetti, Paris, Picard.

Siciliano, Italo, 1968: Les chansons de geste et l'épopée. Mythes, histoire, poèmes, Turin, Società Editrice Internazionale.

Sigebert von Gembloux: siehe die Editionen von Pertz (2) und Witte.

Tischler, Matthias M., 2001: Einharts Vita Karoli. Studien zu Entstehung, Überlieferung und Rezeption, 2 vol., Hannover, Hahn.

TLL: Thesaurus linguae latinae, 1900-, Leipzig, Teubner.

Tremp, Ernst, 1991: Die Überlieferung der Vita Hludowici des Astronomus, Hannover, Hahn.

Van Loey: A. van Loey, ${ }^{7} 1959$, Schönfelds historische grammatica van het Nederlands, Zutphen, Thieme.

Van Rij, Hans, ed. et trad. (niederld.), 1980: Alpertus van Metz, Gebeurtenissen van deze tijd en Een fragment over bisschop Diederik I van Metz, vertaald en ingeleid door H. v. R. met medewerking van Anna Sapir Abulafia, Amsterdam, Verloren.

Van Rij, Hans, 1984: «De Tielse koster, broeder Immo en Alpertus van Metz», in: Ad fontes. Opstellen aangeboden aan prof. dr. C. van de Kieft [...], Amsterdam, Verloren, 1984, 83-94.

Van Rij, Hans, trad. (niederld.), 1999: Alpertus van Metz, Gebeurtenissen van deze tijd, Een fragment over bisschop Diederik I van Metz en De mirakelen van de heilige Walburg, vertaald en ingeleid door H. v. R., Hilversum, Verloren.

Van Winter, Johanna Maria, 1980: «Die Hamaländer Grafen als Angehörige der Reichsaristokratie im 10. Jahrhundert», in: Rheinische Vierteljahresblätter 44, 16-42.

Vita Burchardi: siehe die Edition Waitz.

Waitz, Georg, ed., 1841: «Vita Burchardi episcopi Wormatiensis auctore anonymo», in: Monumenta Germaniae Historica, Scriptores, IV, Hannover, Hahn, 829-846. 
Wirtz, Anna geb. Henningsen, 1971: «Die Geschichte des Hamalandes», in: Annalen des Historischen Vereins für den Niederrhein 173, 7-84.

Witte, Rudolf, ed., 1974: Catalogus Sigeberti Gemblacensis monachi de viris illustribus, Bern, Lang. 
